\title{
Prevalence of myocardial infarction in congestive heart failure patient at emergency room of Rajavithi Hospital
}

\author{
Sumitra Piyanuttapull, Phattarasit Sutarapanakit, Surapun Pongsuthana \\ Rajavithi Hospital, College of Medicine, Rangsit University, Bangkok, Thailand. \\ Correspondence: Sumitra Piyanuttapull. Address: Rajavithi hospital, Bangkok, Thailand. \\ Email: sumitra.piya@gmail.com
}

Received: November 26, 2013 Accepted: June 9, $2014 \quad$ Online Published: July 22, 2014

DOI : $10.5430 /$ jnep.v4n9p111

URL: http://dx.doi.org/10.5430/jnep.v4n9p111

\begin{abstract}
Background: Congestive heart failure is a common condition and along with myocardial ischemia is a global public health problem. In the emergency room, myocardial infarction and uncontrolled hypertension are also found among patients who present with dyspnea from congestive heart failure. Therefore, the researchers chose to study the prevalence of acute myocardial infarction in congestive heart failure patients who presented at the emergency room of Rajavithi Hospital. Improving the screening of patients presenting at the emergency room with congestive heart failure will produce better treatment in the future.
\end{abstract}

Objectives: To determine the prevalence of acute myocardial infarction and prevalence of preserved left ventricular function of patients who present at the emergency room of Rajavithi Hospital.

Method: The study enrolled 91 heart failure patients who presented at the emergency room of Rajavithi Hospital from 1 July 2011 to 31 August 2012. A cross-sectional descriptive study was conducted to determine the prevalence of acute myocardial infarction based on universal definition of myocardial infarction and prevalence of heart failure with preserved left ventricular function. The data were collected from medical records of patients. Statistics and data analysis included percentage, mean and standard deviation using the SPSS version 11.5.

Results: Of the patients, 91 were female (53.8\%), mean age was $66.406 \pm 12.52$ years, main underlying diseases were hypertension and diabetes mellitus. The prevalence of myocardial infarction in this study was $14.13 \%$ (13 patients), and patients with an acute myocardial infarction association were male. The overall body height was higher than in subjects having diabetic mellitus with chronic kidney disease. Patients and CK-MB enzyme level elevations were higher compared with those without myocardial infarction. Mean left ventricular ejection fraction was $44.996 \pm 18.894 \%$, and preserved left ventricular function was 51.6\% (47 patients). Therefore, depressed left ventricular function was significantly found among male patients when compared with the preserved left ventricular function group.

Conclusion: The prevalence of myocardial infarction in congestive heart failure patients was studied. Male patients having diabetes mellitus or chronic kidney disease should be more concerned about myocardial infarction than other population groups. Preserved left ventricular function was associated with a few heart failure cases. Males require earlier detection of the left ventricular function than females. 


\section{Key words}

Congestive heart failure (CHF), Myocardial infarction (MI), Preserved Left Ventricular Systolic function (LVEF)

\section{I ntroduction}

Congestive heart failure is a common condition and a worldwide public health problem. Currently with more than 20 million patients and it continues increasing especially among elderly populations ${ }^{[1]}$. In Thailand, relatively little data is available. However, patients with heart failure are often hospitalized with symptoms of dyspnea. Heart failure patients can be divided into two groups:

1) Heart failure with depressed left ventricular function of the left ventricular (LVEF $<40 \%)$

2) Heart failure with preserved left ventricular function ( $\mathrm{LVEF} \geq 40 \%$ ). Heart failure with normal left ventricular function comprises $40 \%-60 \%$ of all patients with heart failure. In addition, myocardial ischemia is a common problem in public health worldwide. The majority of patients with symptoms of acute myocardial infarction ${ }^{[1]}$ often have problems in terms of chest pain, chest discomfort and angina pectoris. However, patients with myocardial infarction present no angina pectoris and myocardial ischemia can present without symptoms (silent myocardial ischemia or asymptomatic myocardial ischemia).

Patients presenting dyspnea come to the emergency room for many reasons but the most common is myocardial infarction and high blood pressure.

Therefore, the researcher has conducted this study to examine the prevalence of acute myocardial infarction in patients from the emergency room with heart failure. Detecting myocardial ischemia follows the Criteria of Universal Definition ${ }^{[2]}$ in patients with dyspnea at the emergency room and heart failure is diagnosed, according to the Framingham criteria ${ }^{[3]}$. In 1993, Kalon KL and colleagues studied the epidemiology of heart failure patients in the Framingham study (The Epidemiology of Heart Failure: The Framingham Study) and found that heart failure in patients over age 45 years in males occurred in 7.2 cases per 1,000 patients and for female patients 4.7 cases per 1000 patients. The criteria for a diagnosis of heart failure using the Framingham criteria is based on either two major criteria or one major criterion with two minor criteria in the diagnosis of congestive heart failure ${ }^{[3]}$. In 1998, Mihai G and the Board conducted a review of research related to heart failure (Chronic Heart Failure in the United States a Manifestation of Coronary Artery Disease), a randomized multicenter trial published in NEJM from $\mathrm{BC}^{[4]}$. In 1986, a total of 13 issues reported that the prevalence of myocardial infarction was $68 \%$ in patients with congestive heart failure ${ }^{[5]}$. In 1999 , Ramachandran S, et al. conducted a study of 73 patients with heart failure using the Framingham Heart Study (Congestive Heart Failure in Subjects with Normal Versus Reduced Left Ventricular Ejection Fraction Prevalence and Mortality in a Population-Based Cohort) reporting that $51 \%$ of the compression of the left ventricular was normal (normal LVEF) and found more often in women than men ${ }^{[6]}$.

In 2000, Barry FU and colleagues conducted a study (Autopsy in Heart Failure Patients with Sudden Death Results from the Assessment of Treatment with Lisinopril and Survival (ATLAS) trial) to check the operation of acute coronary syndrome among autopsies of patients who died suddenly. The study reported that the conditions of acute coronary syndrome totaled $33 \%$, and those who had vascular disease and coronary heart disease comprised $54 \%$ of all 171 patients examined. The study found that the risk of acute coronary syndrome is common and often asymptomatic, making diagnosis difficult in patients with congestive heart failure especially in patients who died suddenly ${ }^{[7]}$. In 2004, Karen $\mathrm{H}$ and the Board reviewed research data from ten patients with heart failure who experienced a squeeze (Heart Failure with Preserved Left Ventricular Systolic Function, Epidemiology, Clinical Characteristics, and Prognosis). Left ventricular function was usually between $40 \%$ and $71 \%$ and compression of the left ventricles was on average $56 \%{ }^{[5]}$. 
In 2007 and 2012, Kristian T et al. provided new diagnostic criteria shared between ESC/ACCF/AHA/WHF Task Force to determine the criteria for the diagnosis of myocardial infarction (the Universal Definition of Myocardial Infarction). Huxley also shared a similar finding ${ }^{[2,9]}$.

Our study aimed to improve the screening and treatment of patients presenting at the emergency room with heart failure.

\subsection{Primary objective}

To study the prevalence of myocardial infarction in congestive heart failure patients who present at the emergency room of Rajavithi Hospital

\subsection{Secondary objective}

To study the prevalence of heart failure with normal left ventricular function at the emergency room of Rajavithi Hospital.

Definition: Congestive heart failure was diagnosed using the Framingham criteria ${ }^{[3]}$.

\subsection{Major criteria}

The major criteria include: Paroxysmal nocturnal dyspnea, neck vein distension, rales, radiographic cardiomegaly (increasing heart size on chest X-ray film), acute pulmonary edema, third sound gallop, increased central venous pressure ( $>16 \mathrm{~cm}$ water at the right atrium), circulation time $\geq 25$ seconds, hepatojugular reflux, pulmonary edema, visceral congestion or cardiomegaly at autopsy and weight loss $\geq 4.5 \mathrm{~kg}$ in five days in response to treatment of CHF.

\subsection{Minor criteria}

Minor criteria include bilateral ankle edema, nocturnal cough, dyspnea on ordinary exertion, hepatomegaly, pleural effusion, decrease in vital capacity by $33 \%$ from maximal value record and tachycardia (rate $\geq 120$ beats $/ \mathrm{min}$ ).

The diagnosis of heart failure must have at least two major criteria or one major criterion with two minor criteria by which the minor criteria must not have any other medical disease or cause for the minor criteria.

Myocardial infarction was diagnosed using the Universal Definition of Myocardial Infarction and was diagnosed when any of the following occurred ${ }^{[2]}$ :

1) An increase or reduction of cardiac biomarkers (should be troponin) that was detected at least once higher than 99th percentile of the upper reference limit (URL) together with evidence of the occurrence of myocardial ischemia following at least one item listed below.

a) Symptoms of ischemia

b) Changes in the electrocardiogram (ECG) to suggesting new ischemia (new ST-T)

c) Changes or new left bundle branch block, LBBB

d) Caused pathological Q wave in the ECG

e) Imaging evidence of new loss of viable myocardium or new regional wall motion abnormality

2) Sudden unexpected cardiac death, including cardiac arrest with symptom of myocardial ischemia and new ST elevation or new LBBB and fresh thrombus confirmed by coronary angiography or autopsy.

3) Patients are treated by percutaneous coronary interventions (PCI), where the baseline troponin is usually the addition of biomarkers beyond the 99th percentile of the URL. It indicates peri-procedural myocardial necrosis, when more than five times the 99th percentile of the URL. It is a PCI-related myocardial infarction involving stent thrombosis. 
When the PCI patients' baseline troponin does not come down to normal, it may be useful to increase the levels of biomarkers, particularly troponin or CK-MB levels before PCI more than $50 \%$.

4) For patients receiving coronary artery bypass grafting (CABG), the baseline troponin usually shows an increase in the biomarker over the 99th percentile of the URL indicating peri-procedural myocardial necrosis, particularly if more than 10 times. The 99th percentile of the URL is associated with a new pathological Q wave, new LBBB or when injection angiography was found to have occlusion of graft or native coronary artery or imaging, and evidence of loss was found of viable myocardial count as CABG-related myocardial infarction.

5) Pathological results compatible with the diagnosis of acute myocardial infarction ${ }^{[8]}$.

\subsection{Prior myocardial infarction}

The criteria for diagnosis require any of the following ${ }^{[2]}$ :

1) A new pathological $Q$ wave (with or without any symptoms).

2) Detected imaging evidence of a region loss of viable myocardial (thin and without compression) without nonischemic cause.

3) Pathological examination revealing healed or healing myocardial infarction.

\subsection{Detection of the ejection fraction}

The results of the echocardiogram from the examination of the Heart Unit of Rajavithi Hospital evaluated ejection fraction using the Teicholz technique. Heart attack symptoms are checked before in a period not exceeding one year, or after the onset of cardiac arrest in a period not exceeding one month.

1) The ejection fraction of the left ventricular greater than or equal to $40 \%$ to be Preserved Ejection fraction.

2) Values ejection fraction of the left ventricular less than $40 \%$ is considered Depressed Ejection fraction ${ }^{[1,3,5,8]}$.

\section{Method}

The retrospective cross-sectional descriptive study enrolled a total of 102 patients with symptoms of heart failure, who attended the emergency room at Rajavithi Hospital from 1 July 2011 to 31 August 2012.

Selection Criteria Volunteer Program: Inclusion criteria included patients aged 40 years or more, diagnosis of dyspnea or other symptoms diagnosed with heart failure and admitted to the emergency room at Rajavithi Hospital 1 July 2011 to 31 August 2012. Criteria included distinguished volunteers from the project. Exclusion criteria included fatigue or other symptoms or signs of doubt in the diagnosis of heart failure. Diagnostic criteria considered conditions or prohibitions in the diagnosis of myocardial ischemia. Subjects willingly gave their consent to participate and could freely withdraw consent to participate. The selection criteria were provided for patients participating in this research. Patients received a description of the research objectives and explanation of treatment benefits and adverse effects. Patients that participated in the study signed the informed consent form using a code number instead of name data. Tests included Cardiac enzyme Troponin-T, CPK, CK-MB in patients with congestive heart failure to detect myocardial infarction and the result was recorded in the patient record form. Data regarding the heart (echocardiogram) was collected by maintaining compression of the left ventricular (ejection fraction) standard to detect early symptoms of heart attacks in a period not exceeding one year or postnatal heart. Attack in a period not exceeding one month, was noted in the patient record form. After collecting data from all the subjects, statistical analysis using SPSS version 11.5 for window was performed and the findings were presented in the form of a number, percentage, mean, standard deviation, median and range as appropriate. Different data were compared using chi square and independent $T$-test. 


\section{Results}

Of a total of 102 patients, 11 were excluded shown in Figure 1.The majority, 91 patients, were female $53.8 \%$, mean age $66.41 \pm 12.52$ years, mean weight $59.96 \pm 12.21 \mathrm{~kg}$, mean height $156.75 \pm 8.07 \mathrm{~cm}$ and mean BMI $24.42 \pm 4.71 \mathrm{~kg} / \mathrm{m}^{2}$. Systolic Blood Pressure averaged $128.53 \pm 23.88 \mathrm{mmHg}$, while Diastolic Blood Pressure averaged $76.79 \pm 17.29 \mathrm{mmHg}$ for patients with controlled blood pressure criteria. Patients with diabetes or renal disease with blood pressure controlled favorably had scores less than 130/80 $\mathrm{mmHg}$ while patients with other diseases had a threshold blood pressure controlled favorably at less than 140/90 $\mathrm{mmHg}$. In addition, $62.6 \%$ of the co-morbidities of the patients were found to be the main associated factor. Regarding disease, hypertension was found among $64.8 \%$, while $51.6 \%$ had diabetes as shown in Table 1.

In this study of the prevalence of acute myocardial infarction in patients from Rajavithi Hospital 13 subjects had congestive heart failure (14.13\%) while patients with left ventricular function averaged $44.99 \pm 18.89 \%$. Comparing depressed left ventricular function with those without acute myocardial infarction $35.85 \pm 14.98$ vs. $46.52 \pm 19.13$, did not show significant difference. Preserved left ventricular ejection fraction comprised 47 patients (51.6\%). The group with acute myocardial infarction usually tended to have decompressed left ventricular function less than those without acute myocardial infarction $(38.5 \%$ vs. $53.8 \%$ ) but without significance. Comparing patients with heart failure, the acute myocardial infarction was usually associated with men, height of the body, having diabetes, renal failure and CK-MB elevations were significantly higher compared with those without myocardial ischemia. Table 1 shows the baseline characteristics. The group with preserved left ventricular ejection fraction $\geq 40 \%$ and above and the group with the depression of the left ventricular ejection fraction below normal were the two groups that had similar baseline characteristics. Male patients were significantly more likely to have the depression of the left ventricular ejection fraction as shown in Table 2.

Table 1. Baseline characteristics of patients

\begin{tabular}{|c|c|c|c|c|}
\hline & MI (13) & No MI (78) & Mean (91) & $p$-value \\
\hline Age & $65.54 \pm 8.42$ & $66.55 \pm 13.11$ & $66.41 \pm 12.52$ & .829 \\
\hline Female & $2(15.4 \%)$ & $47(60.3 \%)$ & $49(53.8 \%)$ & .003 \\
\hline Weight & $63.96 \pm 10.25$ & $59.29 \pm 12.43$ & $59.96 \pm 12.21$ & .152 \\
\hline Height & $163.69 \pm 6.68$ & $155.59 \pm 7.73$ & $156.75 \pm 8.07$ & .029 \\
\hline Body mass index & $23.82 \pm 3.087$ & $24.52 \pm 4.93$ & $24.42 \pm 4.71$ & .423 \\
\hline Overweight (BMI>23) & $6(46.2 \%)$ & $44(56.4 \%)$ & $50(54.9 \%)$ & .696 \\
\hline Average SBP & $131.62 \pm 29.91$ & $128.01 \pm 22.91$ & $128.53 \pm 23.88$ & .314 \\
\hline Average DBP & $74.77 \pm 18.53$ & $77.13 \pm 17.17$ & $76.79 \pm 17.29$ & .309 \\
\hline Average pulse & $96.23 \pm 19.27$ & $87.19 \pm 21.98$ & $88.48 \pm 21.74$ & .499 \\
\hline Well controlled BP & $7(53.8 \%)$ & $41(52.6 \%)$ & $48(52.7 \%)$ & .932 \\
\hline Hypertension & $11(84.6 \%)$ & $48(61.5 \%)$ & $59(64.8 \%)$ & .107 \\
\hline DM & $10(76.9 \%)$ & $37(47.4 \%)$ & $47(51.6 \%)$ & .049 \\
\hline Dyslipidemia & $3(23.1 \%)$ & $21(26.9 \%)$ & $24(26.4 \%)$ & .771 \\
\hline CKD & $9(69.2 \%)$ & $17(21.8 \%)$ & $26(28.6 \%)$ & $<.001$ \\
\hline CAD & $6(46.2 \%)$ & $23(29.5 \%)$ & $29(31.9 \%)$ & .232 \\
\hline Valvular heart & $1(7.7 \%)$ & $12(15.4 \%)$ & $13(14.3 \%)$ & .463 \\
\hline Average CPK & $481.54 \pm 531.14$ & $92.47 \pm 64.48$ & $148.05 \pm 244.77$ & .315 \\
\hline Average CK-MB & $59.62 \pm 63.43$ & $24.45 \pm 11.72$ & $29.47 \pm 28.41$ & .028 \\
\hline Average LVEF & $35.85 \pm 14.98$ & $46.52 \pm 19.13$ & $44.99 \pm 18.89$ & .825 \\
\hline Preserved LVEF & $5(38.5 \%)$ & $42(53.8 \%)$ & $47(51.6 \%)$ & .304 \\
\hline
\end{tabular}

Note. $\mathrm{SBP}=$ Systolic blood pressure, $\mathrm{BP}=$ Blood pressure, $\mathrm{DBP}=$ Diastolic blood pressure

$\mathrm{DM}=$ diabetes mellitus, $\mathrm{CKD}=$ Chronic kidney disease, $\mathrm{CAD}=$ Coronary artery disease 
Table 2. Preserved LVEF and Depress LVEF

\begin{tabular}{lllll}
\hline & Preserved LVEF (47) & Depress LVEF (44) & Mean (91) & p-value \\
\hline Age & $70.26 \pm 12.03$ & $62.30 \pm 11.81$ & $66.41 \pm 12.52$ & .135 \\
Female & $35(74.5 \%)$ & $14(31.8 \%)$ & $49(53.8 \%)$ & $<.001$ \\
Weight & $59.25 \pm 10.99$ & $60.72 \pm 13.47$ & $59.96 \pm 12.21$ & .222 \\
Body mass index & $24.85 \pm 4.91$ & $23.96 \pm 4.49$ & $24.42 \pm 4.71$ & .395 \\
Overweight (BMI>23) & $26(55.3 \%)$ & $24(56.8 \%)$ & $50(54.9 \%)$ & .156 \\
Well control BP & $24(51.1 \%)$ & $24(54.5 \%)$ & $48(52.7 \%)$ & .740 \\
Hypertension & $34(72.3 \%)$ & $25(56.8 \%)$ & $59(64.8 \%)$ & .121 \\
DM & $27(57.4 \%)$ & $20(45.5 \%)$ & $47(51.6 \%)$ & .253 \\
Dyslipidemia & $13(27.7 \%)$ & $11(25.0 \%)$ & $24(26.4 \%)$ & .774 \\
CKD & $10(21.3 \%)$ & $16(36.4 \%)$ & $26(28.6 \%)$ & .111 \\
CAD & $12(25.5 \%)$ & $17(38.6 \%)$ & $29(31.9 \%)$ & .180 \\
Valvular heart & $8(17.0 \%)$ & $5(11.4 \%)$ & $13(14.3 \%)$ & .441 \\
Average CPK & $128.81 \pm 227.96$ & $168.61 \pm 262.63$ & $148.05 \pm 244.77$ & .442 \\
Average CK-MB & $29.23 \pm 35.67$ & $29.73 \pm 18.12$ & $29.47 \pm 28.41$ & .313 \\
Average LVEF & $60.23 \pm 12.89$ & $28.72 \pm 6.66$ & $44.99 \pm 18.89$ & $<(14.3 \%)$ \\
Myocardial ischemia & $5(10.6 \%)$ & $8(18.2 \%)$ & $13 \%$ & .304 \\
\hline
\end{tabular}

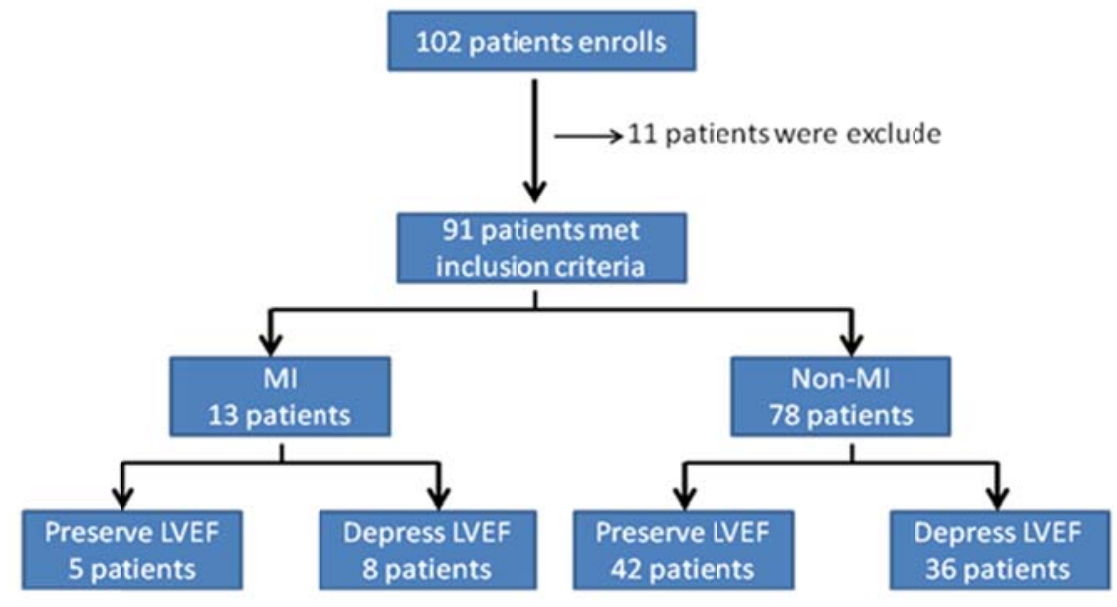

Figure 1. Population of study flow

Table 3. Left Ventricular ejection fraction

\begin{tabular}{lllll}
\hline & MI (13) & No-MI (78) & Total (91) \\
\hline & $<20 \%$ & $1(7.7 \%)$ & $3(3.8 \%)$ & $4(4.4 \%)$ \\
& $20 \%-30 \%$ & $3(23.1 \%)$ & $9(11.5 \%)$ & $12(13.2 \%)$ \\
LVEF* $^{*}$ & $4(30.8 \%)$ & $24(30.8 \%)$ & $28(30.8 \%)$ \\
& $30 \%-40 \%$ & $2(15.4 \%)$ & $10(12.8 \%)$ & $12(13.2 \%)$ \\
& $50 \%-50 \%$ & $2(15.4 \%)$ & $8(10.3 \%)$ & $10(11.0 \%)$ \\
& $50 \%-60 \%$ & $1(7.7 \%)$ & $24(30.8 \%)$ & $25(27.5 \%)$ \\
\hline
\end{tabular}

$* P$-value $=.552$

Comparing the left ventricular ejection fraction in patients in the two groups showed that patients without acute myocardial infarction tended to have good of left ventricular ejection fraction above average, but not significant as shown 
in Table 3. Likely to be Preserved left ventricular ejection fraction (LVEF $\geq 50 \%$ ) was more likely but without significance.

\section{Discussion}

The main cause of heart failure is myocardial ischemia. The causes range from $33 \%$ to $68 \%$ while this study found myocardial infarction at only $14.13 \%$ of patients with valvular heart disease. The heart valve disease can cause heart attack and heart failure without associated acute myocardial infarction. Therefore, the numbers of patients with acute myocardial infarction were fewer in this study than other studies. Data was collected only from patients with myocardial ischemia with myocardial enzyme values. For unstable angina patients, this symptom of acute coronary syndrome causes heart attack stimulation. Therefore, the low number of patients with myocardial infarction may have been caused by fewer patients with myocardial infarction in the study than in other studies. Our study revealed the preserved left ventricular normal at $51.6 \%$, and the depressed left ventricular function to be average, $44.99 \% \pm 18.89 \%$. Previous studies have reported these in the range 40 to $71 \%$, similar to this study. However, when comparing the two groups between myocardial ischemia, our study was more likely to have compression of the left ventricular rates less than those without myocardial ischemia but without significance. This study showed that the left ventricular ejection fraction was impossible to separate from myocardial infarction. Moreover, left ventricle ejection fraction normally can occur cause a heart failure, also known as the preserved systolic function heart failure. Patients in the group with depressed left ventricular ejection fraction were below normal and more commonly found in males significantly. It may be due to males having a risk factor in the occurrence atherosclerosis, coronary artery disease and others. The study found that male patients tended to have myocardial infarction more prevalently. Therefore, patients with depression of the left ventricular ejection fraction were lower in this study. Comparing baseline characteristics of patients between myocardial ischemia and those without myocardial infarction revealed the group with myocardial infarction were males, diabetics, presented renal disease with significant association. The subjects were more likely to be male and have diabetes, a risk factor for atherosclerosis in the coronary arteries. That is more likely to cause acute myocardial infarction. In addition, chronic renal failure is a chronic condition that increases the chance of atherosclerosis. Similarly, the high incidence of myocardial infarction is associated with the risk factors for coronary artery disease, and other related conditions such as high blood pressure. It was higher than in the group with acute myocardial infarction, but it did not differ significantly probably due to the small sample size. Further, it was not the primary objective of this study. The values for myocardial enzyme (CK-MB) was found in the group with myocardial infarction were significantly higher. This was because the diagnosis of acute myocardial infarction is according to the universal definition that requires having the enzymes of myocardial infarction higher than the 99th percentile of the upper normal limit, always involving those with myocardial infarction with the enzyme. In this regard they also found that the two groups differed significantly. The group associated with myocardial infarction had greater body height than the other groups. However, because the number of patients with myocardial infarction comprised only 13 cases, of the deviations from the average could be a possible bias. This research did not intend to study height as the main focus.

\section{Conclusion}

The study investigated the prevalence of myocardial infarction in congestive heart failure patients. Male patients presenting diabetes mellitus or chronic kidney disease should be more concerned about myocardial infarction than other populations. Preserved left ventricular function was held a low association to heart failure. Males should to investigate left ventricular function more than females.

\section{Reference}

[1] Douglas LM. Heart Failure and Cor Pulmonale. Harrison's Principles of Internal Medicine 17th edition. 2008; $1443-1455$. 
[2] Kristian T, Joseph SA, Harvey D, et al. Universal Definition of Myocardial Infarction. Journal of the American College of Cardiology. 2007; 116: 2634-2653.

[3] Bertram P. New Insights into the Epidemiology and Pathophysiology of Heart failure. Journal of the American College of Cardiology. 1993; 22: 6A-13A.

[4] Mihai G and Robert OB. Chronic Heart Failure in the United States: A manifestation of Coronary Artery Disease. Journal of the American College of Cardiology. 1998; 97: 282-289.

[5] Karen H, Karl S, John MM. Heart Failure with Preserved Left Ventricular Systolic Function Epidemiology, Clinical Characteristics, and Prognosis. Journal of the American College of Cardiology. 2004; 43: 317-327. PMid:15013109 http://dx.doi.org/10.1016/j.jacc.2003.07.046

[6] Ramachandran SV, Martin GL, Emelia JB, et al. Congestive Heart Failure in Subjects With Normal Versus Reduced Left Ventricular Ejection Fraction Prevalence and Mortality in a Population-Based Cohort. Journal of the American College of Cardiology. 1999; 33: 1948-1955. http://dx.doi.org/10.1016/S0735-1097(99)00118-7

[7] Barry FU, Kristian T, Paul WA, et al. Acute Coronary Finding at Autopsy in Heart Failure Patients With Sudden Death: Results From the Assessment of Treatment With Lisinopril and Survival (ATLAS) trial. Journal of the American College of Cardiology. 2000; 102: 611-616.

[8] Kenneth D, Alain CS, Gerasimos F, et al. ESC Guidelines for the diagnosis and treatment of acute and chronic heart failure. European Heart Journal. 2008; 28: 2388-2442.

[9] Kristian T, Joseph SA, Harvey D, et al. Third universal definition of myocardial Infarction. Journal of the European Heart Journal. 2012; 33: 2551-2567. PMid:22922414 http://dx.doi.org/10.1093/eurheartj/ehs184 